Case

\title{
A Case of Primary Solitary Pulmonary Plasmacytoma
}

\author{
Hideki Ujiie, MD, Daisuke Okada, MD, PhD, Yuki Nakajima, MD, PhD, \\ Naoyuki Yoshino, MD, PhD, and Hirohiko Akiyama, MD, PhD
}

\begin{abstract}
Most solitary extramedullary plasmacytomas are plasma cell tumors that tend to develop in mucosa-associated lymphoid tissues including the upper respiratory tract. We present a 43-year-old patient who was diagnosed with a solitary plasmacytoma in the lung. Primary plasmacytoma of the lung is exceedingly rare, and the treatment is surgical excision. This malignancy advances to multiple myeloma in a minority of patients. Multiple myeloma is a plasma cell malignancy that typically presents in the bone marrow.
\end{abstract}

Keywords: plasmacytoma, lung, pulmonary, multiple myeloma

\section{Introduction}

Plasmacytoma is a progressive neoplastic disease characterized by diffuse or localized plasmacytic infiltrations and excessive production of monoclonal antibodies (immunoglobulin class G, A, D or E) or immunoglobulinfree $\kappa / \lambda$-light chains.

Extramedullary plasmacytoma (EMP) comprises $3 \%-5 \%$ of all plasma cell neoplasms.

Extraosseous location is extremely rare and may affect any organ. Eighty percent of EMP occurs in the head and neck, and most cases involve the upper aerodigestive tract. ${ }^{1)}$ Primary pulmonary plasmacytoma (PPP) is very rare and is typically found incidentally, during definitive diagnosis of surgically resected specimens.

\section{Case Report}

A 43-year-old man with pulmonary shadows discovered incidentally on chest X-ray was referred to our hospital. Computed tomography scan confirmed the presence

Department of Thoracic Surgery, Saitama Cancer Center, Ina, Saitama, Japan

Received: July 26, 2011; Accepted: August 29, 2011

Corresponding author: Hideki Ujiie, MD. Department of Thoracic Surgery, Saitama Cancer Center, 818 Komuro, Ina, Kitaadachigun, Saitama 362-0806, Japan

Email: hideki_ujiie@hotmail.com

(C)2011 The Editorial Committee of Annals of Thoracic and Cardiovascular Surgery. All rights reserved. of a 10-cm-diameter tumor in the right lung (Fig. 1). The results of serum chemistry measurements, coagulation times, CBC and tumor markers were normal. FDG-PET revealed abnormal uptake of the mass. The patient then underwent a right thoracotomy with right upper lobectomy and partial resection of the middle and lower lung. The pathological diagnosis was a malignant tumor, however, we could not identify the tumor type.

After 3 months of follow-up, the patient exhibited bloody sputum. Chest X-ray detected a mass in the right lung. Computed tomography showed a 55-mm tumor in the epiphrenic region of the right lung (Fig. 2). FDG-PET revealed abnormal uptake of the mass. He underwent a biopsy under CT, and the diagnosis was class V carcinoma. He then underwent a right thoracotomy. Intra operating findings, the tumor was localized right lower lobe. There was enough margin from middle lobe and this case's middle lobe was bigger. We performed right lower lobectomy.

The tumor tissue of the resected specimen exhibited dense infiltrates of monomorphic plasma cells and homogeneous amyloid (HE). The plasma cells exhibited fine chromatin with mild pleomorphism and mitotic activity without evidence of fibrosis or any other inflammatory cell infiltration (Fig. 3A). Immunohistochemistry (IHC) revealed diffuse reactivity with monoclonal $\lambda$-light chain reactivity of the cytoplasm (Fig. 3B). Micro and macroscopic finding showed the section margin was free. However, secondary tumor was rapid growth. Clinically, we think that this is a local recurrence tumor, not a metastatic tumor. 


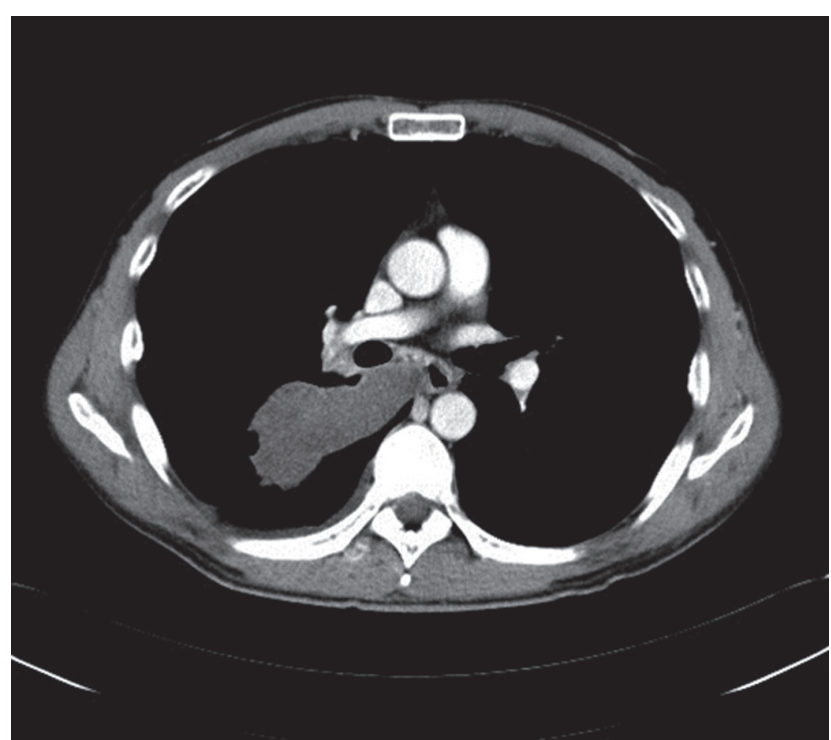

Fig. 1 Computed tomography scan confirmed the presence of a 10 -cm-diameter tumor in the right lung.

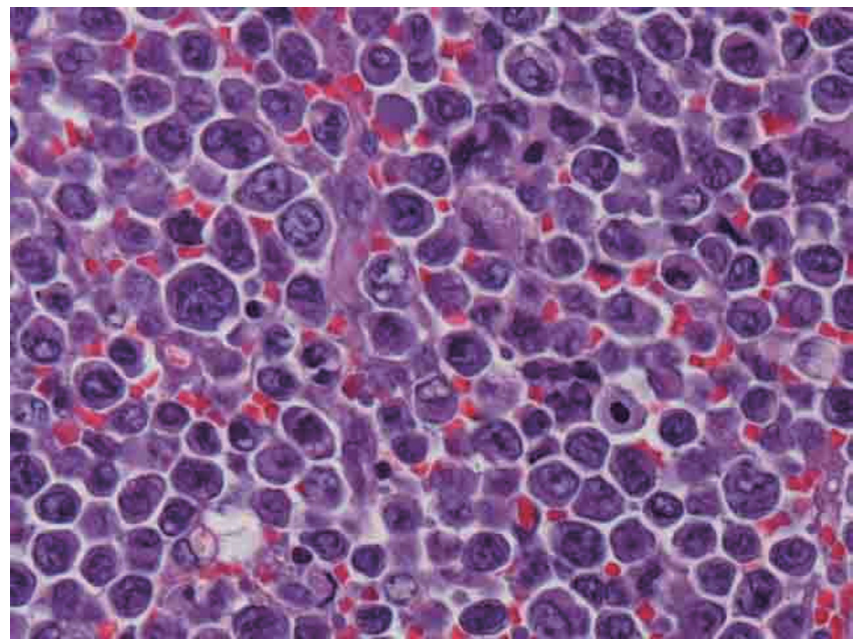

Fig. 3

A: The tumor tissue of the resected specimen exhibited dense infiltrates of monomorphic plasma cells and homogeneous amyloid $(\mathrm{HE} \times 400)$. The plasma cells exhibited fine chromatin with mild pleomorphism and mitotic activity without evidence of fibrosis or any other inflammatory cell infiltration.

B: Immunohistochemistry $(\mathrm{IHC} \times 400)$ revealed diffuse reactivity with monoclonal $\lambda$-light chain reactivity of the cytoplasm.

According to the above findings, the diagnosis of plasmacytoma was confirmed, and a prompt investigation to rule out multiple myeloma was undertaken. Serum immunoglobulin levels were within normal limits. Serum electrophoresis did not reveal the $\mathrm{M}$ component. There was no Bence-Jones protein in the urine. Serum calcium and phosphorus were within their normal ranges. Bone examination revealed no abnormality. A postoperative

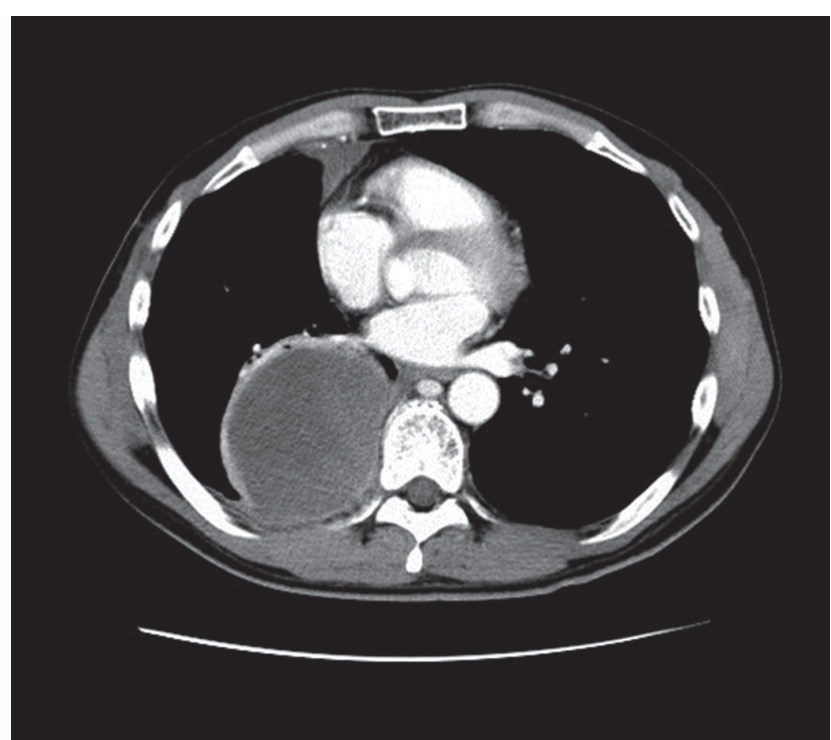

Fig. 2 Computed tomography showed a $55 \mathrm{~mm}$ tumor in the epiphrenic region of the right lung.

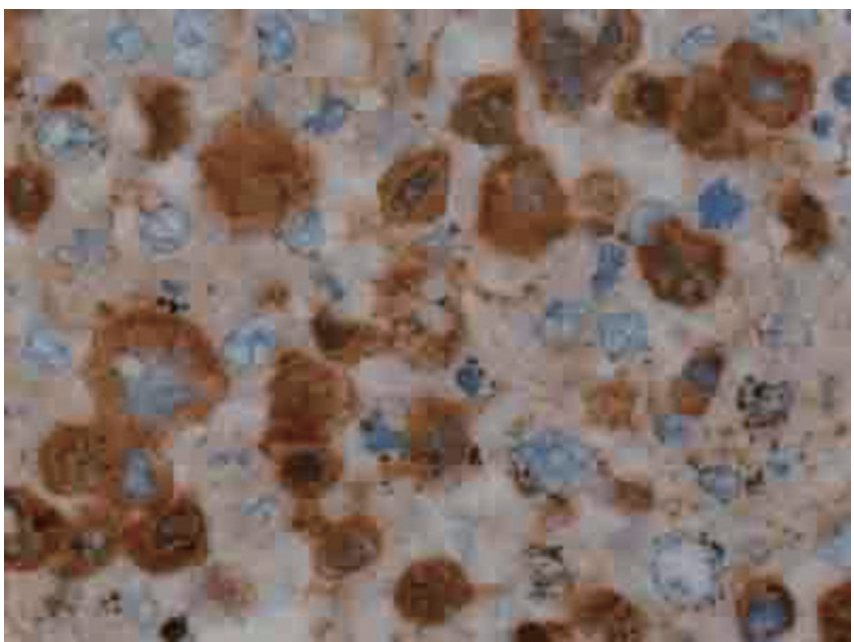

A $\quad B$ sternal bone marrow biopsy specimen showed no abnormalities.

The patient was subsequently diagnosed as having solitary primary pulmonary plasmacytoma.

Post operation course was good. In the chest x-ray, the residual middle lobe was widely expanded.

Six months postoperatively, he continues to do well without evidence of recurrence. 


\section{Discussion and Conclusion}

Multiple myeloma is a systemic monoclonal plasma cell neoplasia that primarily involves the bone marrow. EMP is rare and is observed in only $5 \%$ of all patients with plasmacytoma. PPP is extremely rare, whereas, pulmonary involvement with multiple myeloma is more common. ${ }^{1-3)}$ Although EMP can arise throughout the body, almost $90 \%$ of cases occur in the head and neck, where they typically exhibit a close relationship to mucus membranes.

The presence of clonal plasma cell infiltrates in an extramedullary site can represent multiple myeloma or solitary EMP. About $40 \%$ of the previously reported cases of PPP ultimately developed into multiple myeloma. ${ }^{4}$

To differentiate solitary EMP from multiple myeloma, a bone marrow examination is necessary, and the patient should have fewer than $5 \%$ plasma cells with no dyscrasia and a normal skeletal survey. ${ }^{1)}$

Unlike multiple myeloma, EMP may not exhibit serum $\mathrm{M}$ protein or Bence Jones light chains in the urine. However, up to $25 \%$ of patients with EMP exhibit monoclonal gammopathy (M component).

The male/female ratio is described as 1.4:1, with a median age of 55 years (range, 14-79 years). There is no sexual predilection for primary pulmonary plasmacytoma and most of them are asymptomatic.,5)

Diagnosis is usually made after resection of the tumor. ${ }^{6)}$ Immunohistochemical (IHC) markers are essential to identify monoclonal plasma cells. Negative findings from the postoperative myeloma survey and bone marrow biopsy specimen testing are essential to rule out multiple myeloma.

The pathologic differential diagnosis includes MALT lymphoma with plasma cell differentiation. Extranodal marginal zone lymphoma of the lung exhibits a polymorphous microscopic appearance and cellular remains a hallmark of these lymphomas. The most characteristic cells are small- to medium-size lymphocytes with irregular nuclear outline (centrocyte-like) and plasma cell differentiation is common. However, additional characteristic features include lymphoid follicles with reactive germinal center, lymphoepithelial lesion and fibrosis, in addition to varying proportions of plasma cells, which are either reactive or neoplastic, in the involved tissues.

IHC findings in MALT lymphoma are CD20 +, CD79 $\alpha+$, CD5-, CD10-, CD23-, and CD43-. Our case revealed diffuse monotonous plasma cell infiltration, some with open chromatin pattern, and low mitotic activity without fibrosis or other secondary features, which was totally negative for CD20, CD79a, CD138, and CD56; consistent with diagnosis of plasmacytoma. Gene rearrangement study on paraffin embedded block specimens using PCR revealed the monoclonal band.

Most patients with PPP have been treated with surgery and/or radiotherapy. ${ }^{7)}$ In general, anatomic pulmonary resection is the best treatment of pulmonary plasmacytoma and is performed in $91 \%$ of reported cases. ${ }^{8)}$ There was no evidence of adjuvant systemic therapy. Then we choose observation for this case.

However, when the pulmonary involvement is diffuse, chemotherapy is the best choice. Two previous cases have been treated with melphalan and prednisolone.?)

In $25 \%$ of such cases, it is combined with chemotherapy or radiotherapy and provides a good effect. Longterm results of single chemotherapy/radiotherapy have been significantly worse.

Surgical resection combined with complementary chemotherapy and consecutive, autologous bone marrow transplantation of stem cells could become the treatment of choice, as there is no difference in survival among any of these treatment modalities. The 2-year and 5-year survival rates are $66 \%$ and $40 \%$, respectively. ${ }^{5)}$ Two patients have been reported to survive for 9 years, and two patients have been reported to survive for more than 20 years.

\section{Acknowledgment}

We would like to express our deep appreciation for the support provided for our histopathological examinations by Dr. Yu Nishimura at the pathological/diagnosis department of our hospital. I am deeply grateful to anonymous reviewers.

\section{Disclosure Statement}

I declare that I have no conflict of interest in connection with this paper, other than any noted.

\section{References}

1) Mohammad Taheri Z, Mohammadi F, Karbasi M, et al. Primary pulmonary plasmacytoma with diffuse alveolar consolidation: a case report. Patholog Res Int 2010; 13; 463-5.

2) Gozdziuk K, Kedra M, Rybojad P, et al. A rare case of solitary plasmacytoma mimicking a primary lung tumor. Ann Thorac Surg 2009; 87: e25-6. 
3) Wise JN, Schaefer RF, Read RC. Primary pulmonary plasmacytoma: a case report. Chest 2001; 120: 1405-7.

4) Edelstein E, Gal AA, Mann KP, et al. Primary solitary endobronchial plasmacytoma. Ann Thorac Surg 2004; 78: 1448-9.

5) Koss MN, Hochholzer L, Moran CA, et al. Pulmonary plasmacytomas: a clinicopathologic and immunohistochemical study of five cases. Ann Diagn Pathol 1998; 2: 1-11.

6) Joseph G, Pandit M, Korfhage L. Primary pulmonary plasmacytoma. Cancer 1993; 71: 721-4.

7) Niitsu N, Kohri M, Hayama M, et al. Primary pulmonary plasmacytoma involving bilateral lungs and marked hypergammaglobulinemia: differentiation from extranodal marginal zone B-cell lymphoma of mucosa-associated lymphoid tissue. Leuk Res 2005; 29: 1361-4.

8) [Pulmonary plasmacytoma: about two new cases and review of the literature]. Rev Med Interne 2004; 25 : 591-5. 